\title{
A PROMOÇÃO À SAÚDE CONSTRÓI A VIGILÂNCIA EM SAÚDE: CONTRIBUIÇÕES PARA A EDUCAÇÃO FÍSICA
}

Fabrício Boscolo Del Vecchio

Aguinaldo Gonçalves

\section{Resumo}

No interior do Sistema Único de Saúde, a Vigilância em Saúde estrutura-se como nova área síntese dos segmentos epidemiológico e sanitário. No entanto, diferencia-se deles por incorporar participação ativa dos diversos estratos da sociedade civil nas decisões que lhes dizem respeito. Avança como modelo de atenção à saúde, por reconhecer a determinação social do processo saúde-doença. Nesse âmbito, dialoga com a Educação Física (EF), podendo influenciá-la e ser influenciada por ela; quando da incorporação e aplicação de seus conceitos, agires e fazeres surgem mobilizações intersetoriais que não conduzem só a alterações orgânicas, mas que fomentam motivação e poder coletivo de atuação decorrentes da atividade física (AF) sistematizada. Assim, protagonistas e profissionais de EF podem operar sobre estilos e condições de vida, pela desconstrução do biologicismo exacerbado.

\section{Palavras-Chave}

Promoção à saúde; Atividade física, Vigilância à saúde, Saúde pública/coletiva.

\section{THE PROMOTION TO THE HEALTH CONSTRUCTS THE VIGILANCE IN HEALTH: CONTRIBUTIONS FOR THE PHYSICAL EDUCATION.}

Fabrício Boscolo Del Vecchio

Aguinaldo Gonçalves

\begin{abstract}
Health Surveillance is structured as a new area, which is a synthesis of epidemiological and sanitary segments. Nevertheless it differs from them as it incorporates the active participation of several civil strati in decision affecting them. This is a health care model, recognizing social determination concerning health-disorder-process. Within this context, it dialogues with Physical Education (PE), influencing it and being influenced by it; during its concepts incorporation and application, actions and implementing, intersectorial mobilizations arise, leading not only to organic changes, but also enhance motivation and action collective power connected to systematic physical activity (PA). Thus, PE actors and professionals may operate over life style and conditions, aiming at exaggerate PA biologicism deconstruction.
\end{abstract}

\section{Key-Words}

Health promotion; Physical activity; Health surveillance; Public health. 


\section{INTRODUÇÃO}

A Promoção da Saúde (PS), que partiu inicialmente de enfoque profilático, tem hoje inserção de destaque nas discussões acerca de políticas públicas em saúde (BUSS, 2003). Demonstração disto é o desenvolvimento de encontros internacionais que visam discuti-la sob as mais amplas perspectivas, desde a $1^{\text {a }}$ Conferência Internacional sobre Promoção da Saúde, em Ottawa, 1986, até mais recentemente em Bangkok, 2005, no sexto evento homônimo (os respectivos documentos, e os dos outros anos, podem ser conferidos na íntegra em: www.who.int/healthpromotion/conferences/en). Naquela, PS foi paradigmaticamente reconhecida como o processo de capacitação da comunidade para atuar na melhoria da sua saúde, incluindo maior envolvimento no controle deste processo, com proposição de cinco campos de ação: políticas públicas saudáveis, ambientes favoráveis, reforço da ação comunitária, desenvolvimento de habilidades pessoais e reorientação dos serviços (BRASIL, 2002). Nesta perspectiva de centralidade, a PS passa a ser estratégia mediadora entre indivíduos e seus territórios e visa aumentar a participação destes na modificação dos determinantes das suas saúdes (VICENTIN; PADOVANI; GONÇALVES, 2006).

No entanto, mesmo com mobilização intensa das coletividades nas decisões que thes dizem respeito, cabe lembrar que o Estado tem a responsabilidade de reduzir as iniqüidades, inclusive aquelas relacionadas à saúde (AERTS et al., 2004). A função governamental, assim, dá-se na elaboração e gerência de sistemas de atenção que busquem a eqüidade. O Sistema Único de Saúde, ou simplesmente SUS, surgido das acumulações do Movimento Sanitário e da VIII Conferência Nacional de Saúde, é o modelo nacional áureo no qual objetiva-se esta aproximação igualitária (GONÇALVES, 2006). Nele, fazem-se valer os princípios de universalidade do acesso, integralidade da atenção e descentralização políticoadministrativa.

Consolidado, reforçaram-se suas diretrizes com incorporação de movimentos que o vêm reafirmando democrático; destaca-se o dos agentes comunitários de saúde (ACS), trabalhadores legitimamente integrados às unidades básicas de saúde (UBS), em direção a uma sociedade menos desigual e mais participativa: 91,3\% dos municípios contam com eles, o que vale dizer que quase 100 milhões de brasileiros estão sob suas responsabilidades (GONÇALVES, 2004a). Os ACS estão inseridos no Programa de Saúde da Família (PSF), no qual equipes multidisciplinares formadas, minimamente, por médico, enfermeiro, auxiliar de enfermagem e acompanham $44,4 \%$ de todas as famílias do país (BRASIL, 2004). 
Aspecto que merece ser destacado no interior do SUS é a criação que propiciou de estratégias e reordenamentos, cujas concretizações gradualmente vão inspirando e contribuindo para o desenvolvimento de outras áreas de intervenção e campos do conhecimento. Em tal categoria, situam-se concepções e práticas inovadoras como as referentes ao controle social através de comissões locais ou a composição com o capital privado como instância complementar. Este texto trata de uma dessas iniciativas pioneiras, a Vigilância em Saúde, bem como explora sua possível contribuição para o âmbito da Educação Física. Recorreu-se, para tanto, a conceitos e realizações registrados em recortes da literatura técnica pertinente, ressalvando-se o caráter pluralista e a intenção de abrangência relativa que acaba por conformar.

\section{A PROMOÇÃO À SAÚDE CONSTRÓI A VIGILÂNCIA À SAÚDE}

Antes do SUS, os padrões de assistência à saúde atuavam a partir de demandas espontâneas. Um deles, o sanitarista, esforçava-se em abordar os segmentos populacionais com a estruturação de programas, desenvolvimento de campanhas e organização das vigilâncias sanitária e epidemiológica. Esta se constitui no conjunto de medidas que proporcionam o aprendizado, a detecção e elaboração de diretrizes que assimilem e ajam sobre fatores modificadores da saúde individual ou coletiva, com a finalidade de recomendar e adotar disposições de precaução e controle das enfermidades. Exemplo claro se apresenta na Educação Física (EF), quando, no ambiente escolar, surge a necessidade de procedimento aplicado para bloquear um surto de pediculose ou de infecções fúngicas, que, por meio de indivíduos acometidos, atinja extensos segmentos dessa comunidade (FATARELLI; FRANCO; GONÇALVES, 1997). Nesse caso, a vigilância epidemiológica consiste na atenção dos professores, e do maior contato entre esses e alunos, a partir de referência precisa aos serviços de saúde para cuidado adequado.

Já a sanitária é elenco de medidas que visam preparar, inspecionar e fiscalizar o cumprimento de normas e padrões de interesse relativo a portos, aeroportos e fronteiras, medicamentos, cosméticos, alimentos e saneamento, bem como ao exercício profissional (BARBOSA, 2003). O estudo da situação de águas de piscinas é expressão de seu emprego na área de atividade física, pois já de há muito se aponta que o meio líquido não parece tão saudável para treinamentos e recreação, em função de serem observadas concentrações elevadas de colônias bacterianas e fungos (MENDONÇA; RUFF, 1978). 
Conduta elementar, advogada na EF, traduz-se na verificação da higiene em clubes e academias. Em tal direção, pontua-se que as vistorias são extremamente imprecisas, sem legislação específica e, segundo os próprios funcionários operadores, procedidas apenas em duas perspectivas limitadas, no momento de abertura dos estabelecimentos ou quando ocorrem denúncias por impropriedades que ferem direitos das pessoas (OLIVEIRA, 1997).

Com efeito, os critérios para categorização dos empreendimentos do mercado do fitness, quanto ao estado de limpeza e conforto são inespecíficos e arbitrários, contradizendo, muitas vezes, com a realidade dos espaços disponíveis. No entanto, já existem sugestões numerosas para melhoria das políticas públicas envolvidas na prestação de serviços quanto às práticas corporais. Destaca-se a formação apropriada em nível superior e engenharia correta das instalações destinadas ao público atendido, inclusive aos portadores de necessidades especiais. A inserção das disciplinas da Saúde Coletiva no interior da EF, com estudo do paradigma epidemiológico da produção do conhecimento e manejo das singularidades da educação sanitária, também consiste em estratégia que vem sendo tratada como potencialmente fecunda (GONÇALVES, 2004b).

Desde junho de 2003 conta-se, já inclusive em âmbito institucional, com a concepção e estruturação de nova área síntese dessas duas esferas, a sanitária e a epidemiológica, que é a Vigilância em Saúde - VS (BRASIL, 2003a). Partindo da unificação das referidas segmentações, diferencia-se delas por permitir incorporação de sujeitos da sociedade civil, gestores dos serviços, técnicos e representantes de seções comunitárias organizadas. Em bloco único, tem como desígnios: i) exercitar novo nível de relação com a sociedade, ii) estimular seu poder de decisão, iii) focar a integralidade das pessoas e iv) almejar o atendimento pautado no acolhimento e respeito pelo outro. Como sustentação, em avanço às sistematizações passadas, reconhecem-se a relevância dos componentes objetivos e a determinação social do processo saúde-doença-cuidado (AERTS et al., 2004).

A VS tem como características a intervenção sobre as limitações em saúde, com ênfase naquelas que requerem atenção e acompanhamento contínuos, operacionalização dos conceitos de risco e vulnerabilidade, articulação entre as ações de promoção, prevenção, recuperação e reabilitação, bem como o agir sobre o território, ou seja, o reconhecimento dos espaços físicos segundo a lógica das relações entre condições de vida, saúde e acesso aos serviços (FREITAS, 2003).

Conexões: revista da Faculdade de Educação Física da UNICAMP, Campinas, v. 5, n. 2, p. 59-71, jul./dez. 2007. 
As formas de trabalho são pautadas nas tecnologias de comunicação social, planejamento, programação local e situacional e nas concepções médico-sanitárias. A organização configura-se por meio de políticas públicas saudáveis, gestões intersetoriais, mediações específicas e operações sobre as populações e suas necessidades (BRASIL, 2003b).

Ao superar as dicotomias entre atuações coletivas e individuais, a VS articula-se com a proposta integradora da PS. Deste modo, contemplando necessidades e determinantes sócio-ambientais dos problemas e reforçando a autonomia e o protagonismo, poderá intervir crescentemente sobre realidades brasileiras heterogêneas (TEIXEIRA; PAIM; VILAS BÔAS, 1998).

\section{CONTRIBUIÇÕES PARA A EDUCAÇÃO FÍSICA}

A Atividade Física (AF) é expressão da mobilidade humana que poderia influenciar e ser influenciada pela VS. Materializa-se nas várias esferas da sociedade por fecunda gama de atores com propostas bem diversificadas, desde as ideológico-utilitárias eugenistas e higienistas, até mais recentemente, como égides de luta contra o sedentarismo.

Vale lembrar que, neste cenário, os profissionais da área ainda operam pelas pedagogias do medo e da culpa: a primeira no sentido de se impor estilo de vida ativa pensando na proteção contra eventuais doenças crônico-degenerativas futuras decorrentes da hipocinesia; já a segunda, parte do pressuposto de que aquele que não adota hábitos ditos saudáveis é responsável pela degradação das suas funções orgânicas (QUINT; MATIELLO JR, 1999). Ao ocultarem questões mais amplas abordadas pela VS, como renda, classe social, extração ocupacional, variáveis demográficas, transporte e segurança, inculcam-se encargos exclusivamente pessoais, com destaque à responsabilização da vítima, que tem de recorrer por dever e não por direito à assistência nos moldes privatistas. Neste bojo, encontram-se as políticas de atuação, "marketing" e venda das academias de ginásticas que, baseadas na corpolatria e na culpabilização individual, buscam a venda incessante de serviços (ESTEVÃO; BAGRICHEVSKY, 2004, PALMA; ASSIS, 2005).

As revistas e programas de televisão, amplamente comercializados e consumidos, constituem outro segmento de mercado que age nessa perspectiva,pois, ao apresentarem conselhos e recomendações, apontam caminhos e atitudes a serem seguidos. Ainda, com a divulgação de depoimentos de 
personalidades tomadas como exemplares ditam padrões de beleza, saúde e estética (SERRA; SANTOS, 2003, FIGUEIRA; GOELLNER, 2005).

Exemplo do esforço aplicado no controle das rotinas coletivas advém de países do hemisfério norte. Ela visa à elaboração de objetivos que focam a prevalência da $\mathrm{AF}$ nos diferentes segmentos humanos, $\mathrm{o}$ registro de sua presença no cotidiano das coletividades e caracterizações daquelas mais vulneráveis à exercitação corporal insuficiente (MACERA; PRATT, 2000). Com efeito, nessa perspectiva entende-se AF como manifestação biológica, desprovida de sentido social, a conduzir visão restrita e parcial, calcada nos fatores de risco e opções individuais. Assim, não se consideram as condições de vida como determinadoras de ambos, e reforça-se abordagem equivocada que se difunde no interior da EF. Seus resultados são enviesados na medida em que buscam fomentar implementações que influenciem somente hábitos e ignorem os determinantes objetivos subjacentes. A partir destes registros, apenas de mulheres e homens já mobilizados, estabelecem-se políticas públicas para incremento da AF em escala globalizante. No entanto, os gestores desta vigilância não alertam que, embora eficiente, o sistema não tem interesse de documentar o aumento constante da inatividade (MEROM; BAUMAN; FORD, 2004). Ou seja, enquanto anos se passam, e as ações correspondentes continuam em nível individual, maior a quantidade de sedentários no mundo.

Outrossim, ainda que timidamente, vêm-se contando com iniciativas voltadas a análise das propostas de incentivo aos exercícios físicos. Investigação da realidade brasileira buscou retratar como a prática de AF tem sido estimulada por meio de campanhas (FERREIRA; NAJAR, 2005). Na oportunidade, identificaram-se duas, a "Nacional de Saúde através do Exercício Físico e do Esporte”, desde 1986, e “Agita São Paulo", de 1996, subsidiadas em boa parte com recursos públicos, e que deveriam ser permanentemente avaliadas. De fato, além das volumosas críticas e reservas que vêm se acumulando há longo tempo, mesmo em nosso meio, para com o campanhismo em Saúde (v.g. GONÇALVES, 1981), se um dos objetivos finais destas estratégias é propor a adoção da AF regular, há de se ter indicadores que permitam inferências sobre respectivos impactos. Vale lembrar, ainda, que uma das formas mais pertinentes de estimativa destas diretrizes é a quantificação da AF das populações antes e depois de suas incorporações, procedimento que permanece sendo deixado de lado.

No contexto internacional constatou-se que tais iniciativas aumentam o conhecimento sobre os benefícios de se adotar estilo de vida ativo (STATES et al., 2006), mas não têm efeitos em âmbitos mais amplos, 
retratando pouco incremento do volume de AF realizada após este tipo de intervenção. Ou seja, pontuamse como adequadamente suficientes para aprimoramento da informação acerca da forma de realização da AF e de seus aspectos positivos; no entanto, não se traduzem como consistentes para o vínculo e adesão efetiva (BLAMEY; MUTRIE, 2004).

Reforçam-se, como pontos falhos, a falta de organização, impacto, controle e manutenção das atividades, assim como a ineficiência de conduzir a mudança de padrões, essencialmente agindo nas opções individuais e na noção de demanda espontânea, como faz a maioria dos clubes e academias (CAVILL; BAUMAN, 2004).

Advoga-se, assim, que as situações concretas da vida são pouco modificadas por tais modelos. Com isso, esforços que se concentram em modificar os fatores de risco, sem levar em consideração o contexto social em que se vive, dificilmente promoveriam melhoras significativas no nível de AF. Experiência que foi realizada na cidade de Belo Horizonte, por meio da criação do Índice para a Qualidade de Vida do Lugar Urbano, buscou registrar as condições de vida em relação à AF. Uma das variáveis que o compõe, definida como "Esporte", é estruturada pelos itens "Equipamentos Esportivos", a respeito de área de quadras, campos, clubes, piscinas e congêneres, por habitante, e "Promoções Esportivas", com base no número de eventos esportivos e freqüência de público (FERREIRA; NAJAR, 2005). Outro estudo, agora em condado da West Virginia (EUA), identificou que a implementação de nova pista para caminhada e corrida proporcionou elevação do número de indivíduos fisicamente ativos naquela região. O local foi usado, de modo prioritário, por aqueles que passaram a treinar recentemente, e estes se tornaram mais dependentes dela do que aqueles que o fazem habitualmente (GORDON; ZIZZI, PAULINE, 2004). Assim, planejamentos, programações e ações são construídos pelos protagonistas da situação, com gestões intersetoriais e articulações específicas que operam sobre aspectos subjetivos e objetivos, ao mobilizar maior contingente à ação.

A VS, então, apresenta-se como mediadora para questões mais profundas relacionadas às práticas de AF, que conduzam não só a alterações corporais e orgânicas, mas fomentando maior motivação e poder de atuação decorrentes do exercício. Investigação de seis semanas, com 38 idosos alocados de forma randômica em dois programas de treino, tradicional de força muscular e visando o empoderamento, registrou que aqueles vinculados ao segundo, além de conquistarem melhoras nos testes físicos, também superaram os companheiros quanto ao desejo de obterem melhor aptidão e auto-eficácia para a força, o

Conexões: revista da Faculdade de Educação Física da UNICAMP, Campinas, v. 5, n. 2, p. 59-71, jul./dez. 2007. 
que aumenta a adesão e a manutenção da AF e ganhos subseqüentes (KATULA et al., 2006). Com efeito, fizeram-se valer os princípios da VS no sentido da atenção diferenciada aos vulneráveis, com acompanhamento direcionado à interação social, construído com atividades em pares e desenvolvimento de frentes de trabalho que exigiam maior mediação grupal.

Experiência com saldo positivo envolveu a hidroginástica em nosso meio: a partir de periodização de treze semanas, almejaram-se estímulos para além das conquistas fisiológicas em sessenta mulheres de região carente da cidade de Campinas/SP. Reforçou-se, com exercícios aquáticos, a busca pelo empoderamento daquelas pessoas, ou seja, participação mais enfática acerca das decisões que lhes dizem respeito nos âmbitos social, econômico, cultural e, neste caso, das políticas públicas em direção à motricidade. Além de incrementos na aptidão física, transcenderam-se ganhos significantes em alguns dos domínios de qualidade de vida, propostos pelo WHOQOL-Breve, no sentido de terem sido obtidos:

i) diálogos com diferentes segmentos da sociedade civil, para tratamento de questões surgidas durante a execução do projeto;

ii) democratização da $\mathrm{AF}$, por meio de modalidade habitualmente não acessível a tal estrato, e;

iii) fortalecimento do grupo, a partir da criação de consciência de luta sempre presente entre os protagonistas, nesse caso, como exemplo, para aquisição de piscina disponível, roupas adequadas e transporte (VICENTIN; PADOVANI; GONÇALVES, 2006).

Em função da bilateralidade das relações observadas, os efeitos não se restringiram somente à mudança de estilos de vida: na medida em que elas passaram a construir nova interlocução pela busca de soluções demandadas pelas práticas recém adquiridas, decorrentes da EF, construíam formas de superação das condições materiais limitantes (GONÇALVES, 2006).

Ações no sentido descentralizado e multi-profissional dos agires técnicos, abarcando universalização e integralidade na atenção como, por exemplo, a partir da incorporação dos trabalhadores da EF nas UBS, em contraponto às contratações minimamente necessárias, têm apresentado êxitos. No Reino Unido, observou-se que elevações da ordem de 5\% a 10\% nos esforços vigorosos e moderados semanais podem ser obtidas com abordagem regionalizada dos profissionais, ou seja, atuando em diversos territórios das comunidades, segundo a realidade de cada uma; no entanto, registrou-se naquela oportunidade que entendimento mais completo da interação entre indivíduos e seus ambientes proporcionaria melhoras nos resultados obtidos (HARRISON; ROBERTS; ELTON, 2004).

Conexões: revista da Faculdade de Educação Física da UNICAMP, Campinas, v. 5, n. 2, p. 59-71, jul./dez. 2007. 
Em perspectiva mais próxima, ocorrida em Rio Claro/SP, mulheres vinculadas a programa de AF conduzido em UBS da cidade, treinando 50 minutos duas vezes por semana durante seis meses, conquistaram ganhos na composição corporal, coordenação motora e força (NADAI et al., 2005). Já em nosso meio, foram propostas abordagens com objetivo de se contribuir para o tratamento da hipertensão arterial em Centro de Saúde por meio da AF. Na oportunidade, estruturaram-se dois grupos, com sujeitos alocados randomicamente e, durante oito meses, um deles foi orientado sobre intensidade, freqüência e duração mínima de AF executadas diariamente e o segundo, submetido a esforços físicos programados e supervisionados por professores de EF. Observou-se que, além dos ganhos mais acentuados no último deles, a experiência com a AF foi mais duradoura e significativa (CAMPANE; GONÇALVES; CORREA, 2002).

\section{CONSIDERAÇÕES FINAIS}

Voltando-se de onde se partiu, à medida que o SUS, nestas menos de duas décadas de existência, consolidou-se como o modelo setorial mais bem sucedido de gestão democrática no mundo, pode-se concluir que é amplamente reconhecido que a incorporação de seus princípios norteadores de debate permanente e participação crítica permitem avanços significativos a desafiar a área da Educação Física: trata-se de conquistas de práticas sistemáticas e descentralizadas, discutidas antes e após respectivas intervenções em sessões com durações variadas e conteúdos politemáticos (STATEN et al, 2005). Mas ampliação e aprofundamento nessa direção já é outra história, tratada em outra oportunidade (DEL VECCHIO et al., 2005).

\section{REFERÊNCIAS}

AERTS, D.; et al. Promoção de saúde: a convergência entre as propostas da vigilância da saúde e da escola cidadã. Cadernos de Saúde Pública, Rio de Janeiro, v. 20, n. 4, p. 1020-1028, 2004.

BARBOSA, L. M. M. Glossário de epidemiologia e saúde. In: ROUQUAYROL, Z. M.; ALMEIDA FILHO, N. Epidemiologia e saúde. Rio de Janeiro: MEDSI, 2003, p. 649-690

BLAMEY, A.; MUTRIE, N. Changing the individual to promote health-enhancing physical activity: the difficulties of producing evidence and translating it into practice. Journal of Sports Sciences, Oxford, v. 22, n. 8, p. 741-754, 2004. 
BRASIL. Decreto $\mathrm{n}^{\circ}$ 4726. Aprova a estrutura regimental e o quadro demonstrativo dos cargos em comissão e das funções gratificadas do Ministério da Saúde, e dá outras providências. Diário Oficial da República Federativa do Brasil. Brasília, DF, 9 jun. 2003a.

BRASIL. Ministério da Saúde. As cartas da promoção da saúde. Brasília, 2002. Disponível em $<$ www.saude.gov.br/bvs/conf_tratados.html>, Acesso em: 30 de jan. 2007.

BRASIL. Ministério da Saúde. Secretaria de Vigilância em Saúde. Instrução normativa ${ }^{\circ} 1$. Estabelece procedimentos para elaboração, implementação e acompanhamento da Promoção Pactuada e Integrada de Vigilância em Saúde - PPI-VS. Diário Oficial da República Federativa do Brasil, Brasília, DF, 8 dez. $2003 b$.

BRASIL. Ministério da Saúde, Departamento de Atenção Básica. Saúde da família. Disponível em http://dtr2004.saude.gov.br/dab/atencaobasica.php\#saudedafamilia. Acesso em: 30 jan. 2007.

BUSS, P. M. Uma introdução ao conceito de promoção da saúde. In: CZERESNIA, D.; FREITAS, C. M. Promoção da saúde: conceitos, reflexões e tendências. Rio de Janeiro: FioCruz, 2003, p. 15-38.

CAMPANE, R. Z.; GONÇALVES, A.; CORREA, C. R. A atividade física no controle de hipertensão arterial nos serviços básicos de saúde, a partir do Projeto São Marcos, Campinas, SP. In: SIMPÓSIO DE EDUCAÇÃO FÍSICA E DESPORTOS DO SUL DO BRASIL, 14. Ponta Grossa, 5 a 8 de set. Anais. Ponta Grossa, 2002.

CAVILL, N.; BAUMAN, A. Changing the way people think about health-enhacing physical activity: Do mass media campaigns have a role? Journal of Sports Sciences, Oxford, v. 22, n. 4, p. 771-790, 2004.

DEL VECCHIO, et al. Planejando e estruturando programa de exercícios físicos para intoxicados por mercúrio. Motriz, Rio Claro, v. 11, n.1, 2005, S57-S58.

ESTEVÃO, A.; BAGRICHEVSKY, M. Cultura da "Corpolatria" e body-building: notas para reflexão. Revista Mackenzie de Educação Física e Esporte, Barueri, v. 3, n. 3, p. 13-25, 2004.

FATARELLI, I. F. C.; FRANCO, A .C. S. F.; GONÇALVES, A. Lesões desportivas mais freqüentes. In: GONÇALVES, A. Saúde coletiva e urgência em Educação Física. Campinas: Papirus, 1997, p. 123-134. FERREIRA, M. S.; NAJAR, A. L. Programas e campanhas de promoção da atividade física. Ciência \& Saúde Coletiva, Rio de Janeiro, v. 10, p. 207-219, 2005. (supl.).

FIGUEIRA, M. L. M.; GOELLNER, S. A promoção do estilo atlético na revista Capricho e a produção de uma representação de corpo adolescente feminino contemporâneo. Revista Brasileira de Ciências do Esporte, Campinas, v. 26, n. 2, p. 87-99, 2005.

FREITAS, C. M. A vigilância da saúde para a promoção da saúde. In: CZERESNIA, D.; Promoção da saúde: conceitos, reflexões e tendências. Rio de Janeiro: Fiocruz, 2003. p. 141-159. 
GONÇALVES, A. Intensificações vacinais e sua implementação a partir da administração sanitária local. Folha Médica, Rio de Janeiro, v. 83, n. 4, p. 409-412, 1981.

GONÇALVES, A. Agentes comunitários de saúde: choque de povo. Cadernos de Saúde Pública, Rio de Janeiro, v. 20, n. 6, p. 1771-1772, 2004a.

. Para quê a saúde coletiva na Educação Física? Reflexões de profissional na área há vinte anos. Revista da Educação Física/UEM, Maringá, v. 15, n. 1, p. 89-93, 2004 b.

- A saúde e a atividade física no trabalho, sociedade e meio ambiente: reféns de velhos totens? In: VILARTA, R. et al. (org). Qualidade de Vida e Fadiga Institucional. Campinas: IPES, 2006, p. 7-17.

GORDON, P. M.; ZIZZI, S. J.; PAULINE, J. Use of a community trail among new and habitual exercisers: A preliminary assessment. Preventing Chronic Disease, Atlanta, v. 1, n. 4, p. 1-11, 2004

HARRISON, R. A.; ROBERTS, C.; ELTON, P. J. Does primary care referral to an exercise programme increase physical activity 1 year later? A randomized controlled trial. Journal of Public Health, Oxford, v. 27, n. 1, p. 25-32, 2004.

KATUlA, J. A.; SIPE, M.; REJESKI, W. J.; FOCHT, B. C. Strength training in older adults: an empowering intervention. Medicine and Science in Sports and Exercise, Baltimore, v. 38, n.1, p. 106$111,2006$.

MACERA, C.; PRATT, M. Public health surveillance of physical activity. Research Quarterly for Exercise and Sport, Danvers , v. 71, n. 2, p. 97-103, 2000.

MENDONÇA, C. P.; RUFF, S. D. Estudo das condições sanitárias das águas de piscinas públicas e particulares, na cidade de Araraquara, SP, Brasil. Revista de Saúde Pública, São Paulo, v. 12, n. 2, p. 4549, 1978.

MEROM, D.; BAUMAN, A.; FORD, I. The public health usefulness of the exercise recreation and sport survey (ERASS) surveillance system. Journal of Science, Medicine, and Sport, Mitchell, v. 7, n. 1, p. 32$37,2004$.

NADAI, A. et. Al. Avaliação da aptidão funcional e composição corporal de idosas inseridas em um programa de atividade física moderada. Motriz, Rio Claro, v. 11, n. 1, 2005, S123.

OLIVEIRA, D.T. R. Critérios para avaliação das academias de ginástica: Uma questão de política pública. 1997. Monografia (Graduação) - Faculdade de Educação Física - Universidade Estadual de Campinas, Campinas, 1997.

PAIM, J. S. Vigilância da saúde: dos modelos assistenciais para a promoção da saúde. In: CZERESNIA, D.; FREITAS, C. M. Promoção da saúde: conceitos, reflexões e tendências. Rio de Janeiro: Fiocruz, 2003, p. 161-174.

Conexões: revista da Faculdade de Educação Física da UNICAMP, Campinas, v. 5, n. 2, p. 59-71, jul./dez. 2007. 
PALMA, A.; ASSIS, M. Uso de esteróides anabólico-androgênicos e aceleradores metabólicos entre professores de educação física que atuam em academias de ginástica. Revista Brasileira de Ciências do Esporte, Campinas, v. 27, n. 1, p.75-92, 2005.

QUINT, F. O.; MATIELLO JR., E. O gosto amargo do exercício como remédio nas pedagogias do medo e da culpa. Revista Brasileira de Ciências do Esporte, Campinas, v. 21, n. 1, p. 867-871, 1999.

SERRA, G. M. A.; SANTOS, E. M. Saúde e mídia na construção da obesidade e do corpo perfeito. Ciência \& Saúde Coletiva, Rio de Janeiro, v. 8, n. 3, p. 691-701, 2003.

STATEN, L. K.; et al. Pasos adelante: The effectiveness of a community-based chronic disease prevention program. Preventing Chronic Disease, Atlanta, v. 2, n. 1, p. 1-11, 2005.

STATES, R. A.; et al. Community health education: reaching ethnically diverse elders. Journal of Allied Health, Cambridge, v. 35, n. 4, p. 215-222, 2006.

TEIXEIRA, C. F.; PAIM, J. S.; VILASBÔAS, A. L. SUS, modelos assistenciais e vigilância da saúde. Informe Epidemiológico do SUS, Brasília, v. 7, n. 2, p.7-28, 1998.

VICENTIN, A. P. M.; PADOVANI, C. R.; GONÇALVES, A. Qualidade de vida, empowerment e atividade física em mulheres brasileiras o caso da hidroginástica no Jardim São Marcos, Campinas-SP. In: VILARTA, R. et al (Org.). Qualidade de Vida e Fadiga Institucional. Campinas: IPES, 2006, p. 255-270. 


\section{Fabrício Boscolo Del Vecchio}

Faculdade de Educação Física - UNICAMP

\section{Aguinaldo Gonçalves \\ Faculdade de Educação Física - UNICAMP}

\section{Referência do artigo:}

\section{ABNT}

VECCHIO F. B., GONÇALVES, A. A promoção à saúde constrói a vigilância em saúde: contribuições para a educação física. Conexões, v. 5, n. 2, p. 59-71, 2007.

\section{APA}

Vecchio, F. B., \& Gonçalves, A. (2007). A promoção à saúde constrói a vigilância em saúde: contribuições para a educação física. Conexões, 5(2), 59-71.

\section{VANCOUVER}

Vecchio $\mathrm{FB}$, Gonçalves A. A promoção à saúde constrói a vigilância em saúde: contribuições para a educação física. Conexões, 2007, 5(2): 59-71. 